\title{
Near-field radiation pattern distortion of antenna attached to wall in through-the-wall radar imaging
}

\author{
B. Cetin, H.-R. Benedickter, and P. Leuchtmann \\ Institute for Electromagnetic Fields and Microwave Electronics, ETH Zurich, Switzerland \\ Correspondence to: B. Cetin (bora.cetin@ifh.ee.ethz.ch)
}

\section{Introduction}

Through-the-wall radar imaging (TWRI) has become subject of growing interest in the recent decade. It is supposed to be used for law-enforcement purposes during surveillance, reconnaissance and rescue missions (Ferris et al., 1999; Baranoski, 2008; Amin, 2011). Radar systems in general are based on the detection of reflected waves caused by scattering objects. According to shape and amplitude of the reflection, the measurement data are processed into a tomographic image. When the signal is propagating through-thewall (TTW), the reflected signal's original shape and amplitude are altered. For the reconstitution of the reflection of interest (Thajudeen et al., 2011), the distortion causing signal path needs to be known regarding to its structure and electromagnetic material properties. Otherwise the image will be degraded and defocused (Li et al., 2010; Chang et al., 2010).

In many studies the properties of different kinds of construction material have been investigated (Muqaibel, 2003; Stavrou et al., 2003). Due to the natural origin of some materials (e.g. wood and brick), natural conditions (e.g. moisture) and not standardized material mixtures (e.g. concrete), published material values can differ significantly. To overcome this uncertainty in material parameters, on-site material extraction methods have been proposed (Ahmad et al., 2007; Jia et al., 2011). These methods are based on the single layer homogeneous wall model and have a limitation in accuracy. In a realistic inhomogeneous multilayer wall, however, multipath propagation and anisotropic wall characteristics influence the detected value of material properties.

Reduction of clutter caused by ceiling, floor and the back wall can be achieved by placement of the antenna close or even directly on the walls themselves (Jia et al., 2011). At the same time the wall penetrating electromagnetic wave's power density will be higher, resulting in a more sensitive sensing of reflections (Farwell et al., 2008). In addition, an- other uncertainty in the system gets omitted: the distance between wall and antenna (Wang et al., 2006). By the placement of the antenna directly on the wall and the decrease of the distance between antenna and target into the near field distance jointly with the use of large imaging apertures, the resolution of the imaging system can be optimized (Sheen et al., 2007).

This work analyzed the distortion of the radiation pattern when the antenna is placed on wall. Sensitivity analysis were made to investigate the impact of imprecise knowledge of material parameters on the radiation pattern. The principle setup is explained in Sect. 2. The used components including antenna and the examined walls are presented in Sect. 3. In Sect. 4 the simulation techniques are explained, whereas Sect. 5 shows the measurement setup. The results are presented and discussed in Sect. 6, followed by the conclusions in Sect. 7.

\section{Principle setup}

The radiation pattern is a characteristic parameter of an antenna. It describes the radiated electromagnetic wave properties (e.g. signal strength, phase, polarization) in dependence of the radiation direction. For obtaining the radiation pattern, a relative rotation between the antenna under test (AUT) and the monitoring system is required. In a simulational environment field monitors can spot the field strength on a sphere around the AUT. In measurements, the AUT and the probe antenna need to be rotated relatively against each other as drafted in Fig. 1. There are two technical implementations of this principle (Balanis, 2005). The first one is based on the rotation of the AUT around its own axis in azimuth plane. In the second method, the probe antenna is moved circularly around the AUT. Both methods are suitable for determining the radiation pattern of the antenna when put directly on the 


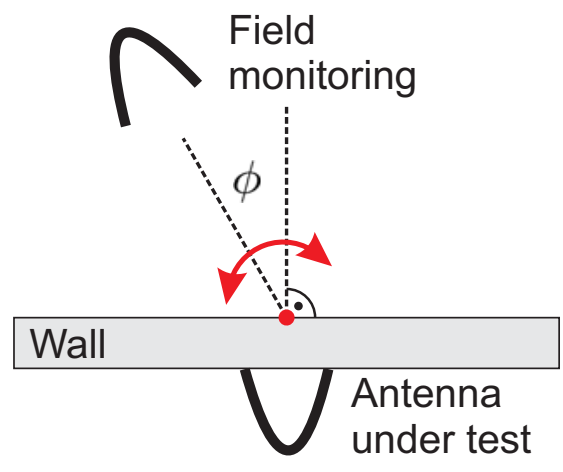

Fig. 1. Principle of obtaining the radiation pattern in simulations and measurements. It is based on the relative rotation $\phi$ between the antenna under test (AUT) and the monitoring system (field monitor in simulations, probe antenna in measurements). Here, the setup shows the antenna put directly on a wall.

wall. The method was chosen in dependence of the circumstances as described in Sect. 5.

Two datasets for each the simulations and the measurements are required for determining the influence of a wall on an antenna's radiation pattern: the first dataset is for determining the non-distorted radiation pattern of the AUT in free-space. The second one is for the distorted radiation pattern that results from the placement of the antenna directly on the wall. By comparison of both datasets, the distortion of the radiation pattern and the damping through-the-wall (TTW) $\Delta s_{21}$ caused by the wall can be quantified

$\Delta s_{21}=s_{21, \text { withwall }}-s_{21, \text { freespace }}[\mathrm{dB}]$.

\section{Components}

The distortion of an antenna's radiation pattern is being analyzed when the antenna is placed directly on a wall. The investigations include simulations and measurements in the frequency range $0.7-10.0 \mathrm{GHz}$ as proposed for TWRI.

\subsection{Antenna}

The used antenna is the ETS-Lindgren 3164-08, a nonresonant ultra-wideband (UWB) antenna based on the quadridged horn design. It is dual-polarization capable and operational in the frequency range of interest. This antenna's reactive part within the near-field (NF) region is situated in the coupling region between the coaxial input and the ridges. As it vanishes within one wavelength, no reactive NF is present at the antenna's mouth and therefore only very limited mutual coupling between antenna and wall is possible (Seybold, 2005).
Table 1. Material parameters at $f=1.75 \mathrm{GHz}$ derived from rectangular waveguide measurements and used for the simulations. The sensitivity analysis was carried out at $2.0 \mathrm{GHz}$.

\begin{tabular}{|c|c|c|}
\hline Material & $\varepsilon_{\mathrm{r}}$ & $\tan \delta$ \\
\hline Wood & 1.81 & 0.077 \\
\hline Sensitivity analysis & $\begin{array}{lll}1.6 & 1.7 & 1.8\end{array}$ & $\begin{array}{lll}0.06 & 0.07 & 0.08\end{array}$ \\
\hline Brick & 3.49 & 0.005 \\
\hline Sensitivity analysis & \multicolumn{2}{|c|}{ Spacing between bricks $g: 1 \mathrm{~cm} 2 \mathrm{~cm}$} \\
\hline Sand lime brick & 5.02 & 0.035 \\
\hline Insulating foam & $\approx 1$ & $\approx 0$ \\
\hline
\end{tabular}

\subsection{Wall materials}

The first class of material investigated is the homogeneous dielectric slab. Representatively for this class of material, glued laminated timber made of wood with the size of Thickness (T) $4.0 \mathrm{~cm} \times$ Length (L) $200 \mathrm{~cm} \times$ Width (W) $60 \mathrm{~cm}$ is chosen. Putting three specimens together yield in a $180 \mathrm{~cm}$ wide wooden slab. The second class of material is inhomogeneous brick. The size of the used bricks is $\mathrm{L} 29.0 \mathrm{~cm} \times \mathrm{W}$ $17.5 \mathrm{~cm} \times$ Height (H) $19.0 \mathrm{~cm}$ (Swiss Modul 030419, Fig. 2). The third wall is a typical Swiss multilayer inhomogeneous exterior wall with a total thickness of $60 \mathrm{~cm}$. It consists of three layers, namely sand lime brick (Creabeton 510604, L $25.0 \mathrm{~cm} \times \mathrm{W} 14.5 \mathrm{~cm} \times \mathrm{H} 19.0 \mathrm{~cm})$, insulating foam $(\mathrm{T}$ $15.0 \mathrm{~cm}$ ) and brick (Swissbrick, L $29 \mathrm{~cm} \times \mathrm{W} 17.5 \mathrm{~cm} \times \mathrm{H}$ $19 \mathrm{~cm})$. A comparison of Fig. 2 and Fig. 3 shows that the internal structure of the used bricks are almost identical. Before measuring the material parameters at $f=1.75 \mathrm{GHz}$ as listed in Table 1, the stones and bricks were dehumidified in an oven. In dependence of the permittivity $\varepsilon_{\mathrm{r}}$, the wavelength of the electromagnetic wave reduces to

$\lambda=\frac{\lambda_{0}}{\sqrt{\varepsilon_{\mathrm{r}, \mathrm{eff}}}}=\frac{\lambda_{0}}{\sqrt{\varepsilon_{\mathrm{r}}}} \sqrt{\frac{V_{\text {total }}}{V_{\text {material }}}}$

when propagating through material. The effective permittivity of an inhomogeneous material distribution can be calculated by the material's permittivity and the ratio of the material volume to total volume (see Fig. 2), provided that the cavities are much smaller than the wavelength.

\section{Simulation technique}

Simulations were carried out to cross-check with the measurements. As simulations are virtual, geometries and material parameters can be changed in the setup for sensitivity analysis purposes. Scenarios that cannot be measured experimentally still can be simulated, enabling investigations of extreme scenarios. The simulations were carried out using the Finite-Element-Method (FEM) field simulator HFSS (High 


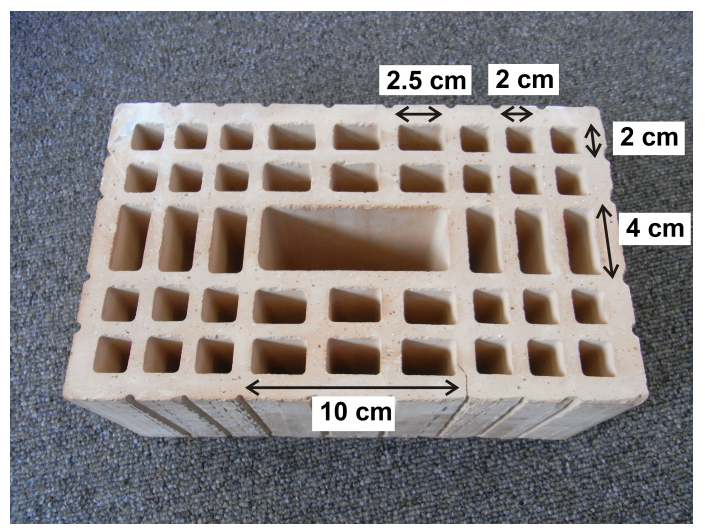

Fig. 2. Cross-section of the brick used in these investigations. Geometry is almost identical to brick used in the multilayer wall (see Fig. 3). The cavities cause the decrease of the effective permittivity.

Frequency Structure Simulator). For this, the antenna's geometry and the different kinds of wall were implemented in the programme. The use of the FEBI (Finite Element Boundary Integration) boundary condition helped reducing the computational volume (Edgar, 2011) for the simulation of the single antenna. For overcoming memory shortcomings for the simulation of the whole structure including antenna and wall, the FEBI and Datalink (a technique for separating the computational space into parts) functions were used jointly, facilitating the computational separation of antenna and wall and retaining the distance between them close to zero at the same time.

\section{Sensitivity analysis}

To evaluate the effects of differing material parameters in the case of wooden slab, a sensitivity analysis was carried out at $f=2.0 \mathrm{GHz}$ with three different values for the permittivity $\varepsilon_{\mathrm{r}}$ and three different values for the loss tangent $\tan \delta$, respectively (see Table 1). For this, the damping $\Delta s_{21}$ TTW defined in Eq. (1) was determined for all nine material properties combinations and compared with the reference $\left(\varepsilon_{\mathrm{r}}=1.7\right.$, $\tan \delta=0.07)$. In the case of brick wall, a sensitivity analysis was performed on the horizontal and vertical spacings $g$ between the bricks. Two different values (see Table 1) have been used to evaluate the impact of brick spacing on the radiation pattern.

\section{Measurement setup}

The radiation pattern of the AUT was measured in different antenna configurations. These are described in this section and the specific setup parameters are summarized in Table 2. The probe antenna for recording the electromagnetic field strength on a circle around the AUT in azimuth plane is of the same model as the AUT itself (Sect. 3.1). All radia-

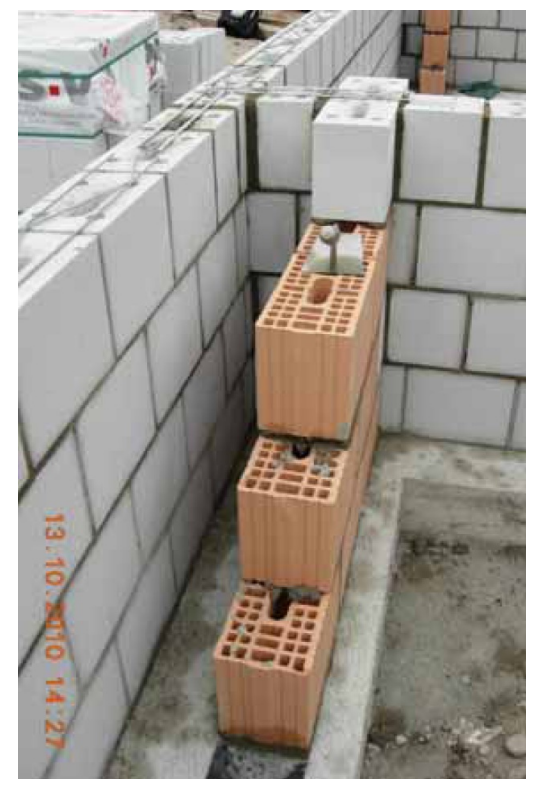

Fig. 3. Cross-section of the multilayer wall on the open-air test site Witaumatte (Thun, $\mathrm{CH}$ ). The used materials are sand lime brick (left), brick (right) and insulating foam in between (not visible in this picture).

tion patterns were recorded in the four possible polarization directions (co-polarizations $\mathrm{HH}$ and $\mathrm{VV}$, cross-polarizations $\mathrm{HV}$ and $\mathrm{VH}$; the letters denote the polarization of the AUT and probe antenna, respectively).

The measurements were carried out in the frequency domain, using an Agilent 8510C Vector Network Analyzer (VNA). The calibration was applied to the connection interface between coaxial cable and antenna. Therefore, the measurements include all effects due to frequency dependency of the antennas. However, they are cancelled by the subtraction of both datasets as described in Sect. 2 .

\subsection{Free-standing antenna in anechoic chamber}

The radiation pattern of the AUT in free-space configuration was recorded in the anechoic chamber. It serves as a reference for evaluating the distorted radiation patterns of the AUT when put directly on different kinds of wall. The relative rotation between the AUT and the probe antenna was achieved by rotating the AUT around its own axis in azimuth plane. Due to the use of a robotic device, the adjusted azimuth angle $\phi$ is very precise.

\subsection{Antenna on homogeneous wooden slab and inhomogeneous brick wall in anechoic chamber}

The distorted radiation patterns of the AUT when put on homogeneous wooden slab and inhomogeneous brick wall (see Fig. 4) was recorded in the anechoic chamber. For this, the 
Table 2. Geometrical setup parameters for both the simulations and the measurements. The simulations provide data for all required distances and for $\phi=\left[-180^{\circ}, 180^{\circ}\right]$ with $\Delta \phi=1^{\circ}$.

\begin{tabular}{|c|c|c|c|c|}
\hline $\begin{array}{l}\text { Antenna } \\
\text { configuration }\end{array}$ & $\begin{array}{l}\text { Distance AUT } \\
\leftrightarrow \text { probe antenna }\end{array}$ & $\begin{array}{l}\text { Recorded azimuth } \\
\text { angle interval } \phi\end{array}$ & $\begin{array}{c}\text { Increment in } \\
\text { azimuth angle } \Delta \phi\end{array}$ & $\begin{array}{l}\text { Recorded frequency } \\
\text { spectrum } f\end{array}$ \\
\hline $\begin{array}{l}\text { Free-standing } \\
\text { (indoor) }\end{array}$ & $\begin{aligned} d_{1} & =1.50 \mathrm{~m} \\
d_{2} & =2.70 \mathrm{~m} \\
d_{3} & =4.14 \mathrm{~m}\end{aligned}$ & $\begin{array}{c}{\left[-178^{\circ}, 180^{\circ}\right]} \\
{\left[-178^{\circ}, 180^{\circ}\right]} \\
0^{\circ}\end{array}$ & $\begin{array}{l}2^{\circ} \\
2^{\circ} \\
-\end{array}$ & $\begin{array}{l}0.7-10.0 \mathrm{GHz} \\
0.7-10.0 \mathrm{GHz} \\
0.7-10.0 \mathrm{GHz}\end{array}$ \\
\hline $\begin{array}{l}\text { On wooden slab } \\
\text { (indoor) }\end{array}$ & $\begin{array}{l}d_{1}=1.50 \mathrm{~m} \\
d_{2}=2.70 \mathrm{~m}\end{array}$ & $\begin{array}{c}{\left[0^{\circ}, 70^{\circ}\right]} \\
{\left[-35^{\circ}, 70^{\circ}\right]}\end{array}$ & $\begin{array}{l}5^{\circ} \\
5^{\circ}\end{array}$ & $\begin{array}{l}0.7-10.0 \mathrm{GHz} \\
0.7-10.0 \mathrm{GHz}\end{array}$ \\
\hline $\begin{array}{l}\text { On brick wall } \\
\text { (indoor, Fig.4) }\end{array}$ & $d_{1}=1.50 \mathrm{~m}$ & {$\left[-25^{\circ}, 60^{\circ}\right]$} & $5^{\circ}$ & $0.7-10.0 \mathrm{GHz}$ \\
\hline $\begin{array}{l}\text { On multilayer wall } \\
\text { (outdoor, Fig.6) }\end{array}$ & $\begin{aligned} d_{1} & =1.50 \mathrm{~m} \\
d_{4} & =3.00 \mathrm{~m}\end{aligned}$ & $\begin{array}{c}{\left[-60^{\circ}, 60^{\circ}\right]} \\
0^{\circ}\end{array}$ & $\begin{array}{c}10^{\circ} \\
-\end{array}$ & $\begin{array}{l}0.7-4.3 \mathrm{GHz} \\
0.7-4.3 \mathrm{GHz}\end{array}$ \\
\hline
\end{tabular}

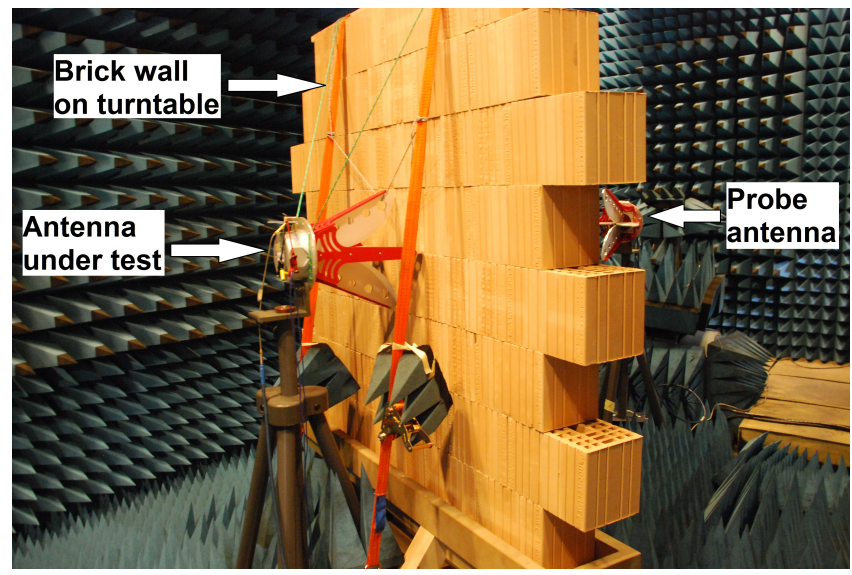

Fig. 4. Measurement setup of antenna placed on brick wall in position A (see Fig. 5). The structure comprising of wall and AUT is being rotated around its axis and the radiation pattern is recorded with the probe antenna. Measurements were carried out inside an anechoic chamber. The setup for the wooden slab is accordant.

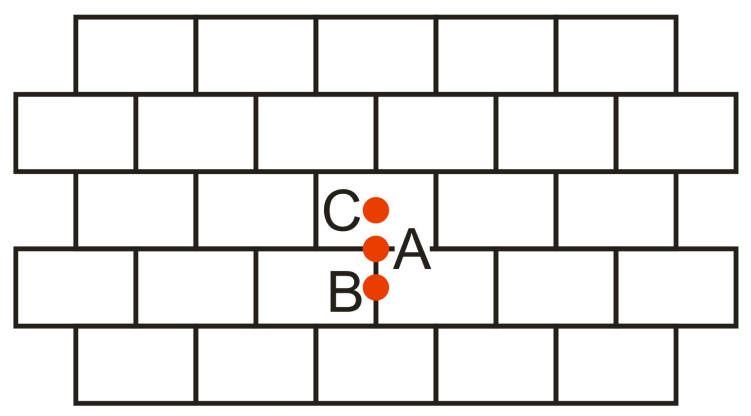

Fig. 5. Measurement positions for AUT on the brick wall. Two rows are omitted at the bottom and one row at the top.

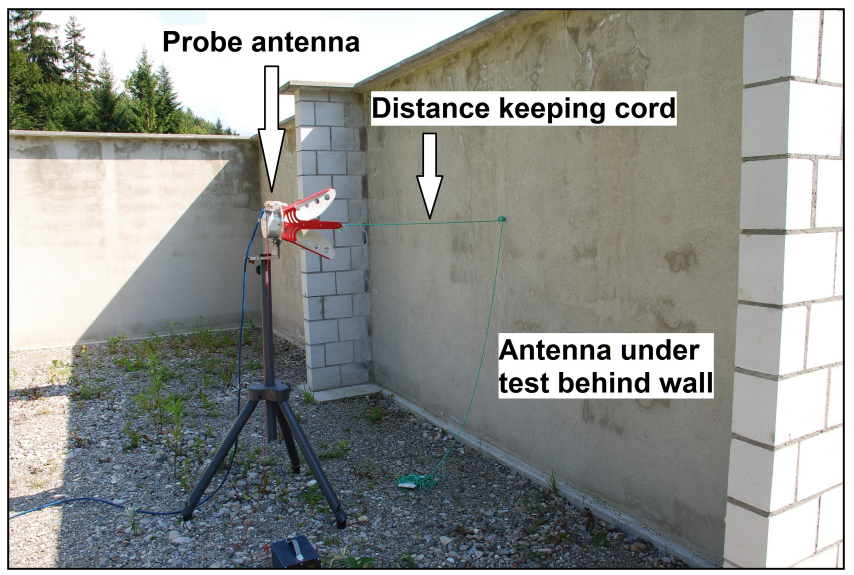

Fig. 6. Measurement setup of antenna on multilayer wall on the open-air test site Witaumatte (Thun, $\mathrm{CH}$ ). The AUT is being placed directly on the wall. The radiation pattern is recorded with a probe antenna that is moved on a circle around the wall (distance keeping cord).

walls were erected on a table standing on a turntable inside the anechoic chamber. The AUT was fixed on the walls. By rotating this structure around its own axis, the distorted radiation pattern was recorded with the probe antenna being on a fixed spot. The use of an angle metre in connection with the special construction of the table helped keeping the step sizes $\Delta \phi$ very precise. Due to a mistake in the reference $\phi_{\text {offset }}$, the data are recorded with an offset in angle that is constant for all $\phi$. This mistake was corrected in Sect. 6.1 in case of the homogeneous wooden slab. Due to the brick's inhomogeneous profile (Fig. 2), transmissions TTW are sensitive to the location of measurement which makes corrections senseless as other effects are very much dominating the measurements. For quantification of the sensitivity, radiation pattern measurements have been carried out at the three differing positions on the brick wall as indicated in Fig. 5. 


\subsection{Antenna on inhomogeneous multilayer wall at open-air test site}

In the case of the inhomogeneous multilayer wall at the openair test site in Thun $(\mathrm{CH})$, the probe antenna was moved circularly around the AUT as shown in Fig. 6. Even though the same angle metre was used here as well, a less precise adjustment of the azimuth angle $\phi$ is being expected. A deviation of a few degrees for each position is realistic. Due to being in a realistic environment, parasitic effects and disturbance sources can interfere with the measurements. An attenuator was used for suppressing these interferences (and parasitically the signal). A 10-Watt amplifier was used to raise the signal. This limited the frequency range to $0.7-4.3 \mathrm{GHz}$.

\section{Results and discussion}

For the calculation of the net damping $\Delta s_{21}$ caused by the multilayer wall, free-space measurements and measurements on the wall at corresponding distances and the same VNA setting were executed. In this case the only change in the configuration is the presence of the wall and $\Delta s_{21}$ can be calculated by subtracting the results of both measurements from each other as shown in Eq. (1).

\subsection{Homogeneous wooden slab}

In contrary to the perfectly symmetrical simulational setup, inaccuracies lead to an offset azimuth angle $\phi_{\text {offset }}$ in the measurement setup. This offset was quantified by the identification of the symmetry centre of the radiation pattern recorded at the distance $d_{2}=2.70 \mathrm{~m}$. Its value is $\phi_{\text {offset }} \approx 2^{\circ}$, and the results were corrected accordingly. Simulated and measured damping $\Delta s_{21}$ in the azimuth plane is plotted in Fig. 7 for $f=1.0 \mathrm{GHz}$ and the distance $d_{2}=2.70 \mathrm{~m}$ between AUT and observation point. Due to the perfect symmetry in the field simulator, the simulation results for both polarizations are symmetric. In the case of an infinite slab, perpendicular (VV) and parallel (HH) polarization directions within the plane of incidence fall together at normal incidence and show the same transmission characteristics. As the simulation results intercept at $\phi=0^{\circ}$, the effects of the slab edges are negligible at this angle. In contrary, for the measurements there is a difference of $\approx 0.15 \mathrm{~dB}$ between both polarizations at normal incidence, resulting from positioning imprecisenesses like inclination of the slab, adjustment and torsion of the antenna. For these reasons the recorded radiation pattern is affected irreversibly. With increasing azimuth angle both simulation and measurement data for the VV polarization show corrugation behaviour, in contrast to the HH polarization data that stays smooth. This results from the antenna's radiation pattern that is broader in the cut perpendicular to the excitation (ETS-Lindgren, 2010): as the data was recorded in azimuth plane, this complies with the VV data. Due to broader main lobe, the propagating wave inter- acts with the edges of the slab, causing wave interference in the observation point. The damping is almost constant for $\phi<40^{\circ}$. The wave propagating TTW gets attenuated only in the normal direction of the slab. Therefore, the longer propagation path of the electromagnetic wave described by Snell's law does not lead to bigger damping values $\Delta s_{21}$. Simulations and measurements are in good agreement for $\phi<45^{\circ}$. If the angle increases further, disturbing effects caused by the slab's edges dominate the results. Minimizing these effects are only possible by using a bigger wooden slab in simulations and measurements.

The results of the accomplished sensitivity analysis are plotted in Fig. 8. The deviation in damping $\Delta s_{21}$ is shown in dependence of $\varepsilon_{\mathrm{r}}$ (x axis) and $\tan \delta$ (coloured bars). The values $\varepsilon_{\mathrm{r}}=1.7$ and $\tan \delta=0.07$ are considered as reference. The maximum deviation for the values under study is approximately $0.25 \mathrm{~dB}$, this means the relative uncertainty is about $3 \%$. If the assumed $\varepsilon_{\mathrm{r}}$ is correct, the maximum deviation caused by a wrong $\tan \delta$ is about $0.2 \mathrm{~dB}$ (relative uncertainty $2 \%$ ), in case the $\tan \delta$ is known and $\varepsilon_{\mathrm{r}}$ is unknown, the maximum deviation is about $0.1 \mathrm{~dB}$ (relative uncertainty $1 \%)$. The deviation due to an overestimated $\varepsilon_{\mathrm{r}}$ gets partially compensated with an underestimated $\tan \delta$, and vice versa accordingly. In this case the deviation is limited to $0.1 \mathrm{~dB}$ (relative uncertainty $1 \%$ ).

As a recapitulation it can be said that the distortion of the antenna's radiation pattern is limited to damping $\Delta s_{21}$ for measurement angles $\phi<45^{\circ}$. Due to the symmetry and homogeneous character of the material, this does not only apply to the azimuth plane but also to the inclination plane: $\mathrm{HH}$ simulations and measurements in the azimuth plane can also be interpreted as VV in the inclination plane. The good agreement of simulations and measurement show that transmission through a homogeneous slab is predictable. The sensitivity analysis shows that the quantified deviations in the result are smaller than the deviation in the material parameter. While the relative uncertainty of the permittivity $\varepsilon_{\mathrm{r}}$ is about $6 \%$ and that of the loss tangent $\tan \delta$ is about $14 \%$, the combined maximum consequence in $\Delta s_{21}$ is just $3 \%$. If one of the two material parameters $\left(\varepsilon_{\mathrm{r}}\right.$ and $\left.\tan \delta\right)$ is overestimated and the other one is underestimated, this leads to a partial compensation. At the same time the deviations are quite small and do not affect the radiation pattern dramatically. If the importance of the signal amplitude is of minor interest, the deviation due to wrong material parameters within the range of this investigation can be neglected.

\subsection{Inhomogeneous brick wall}

In the first step, the measured data at all three positions on the wall as shown in Fig. 5 were compared regarding to the main radiation direction $\phi=0^{\circ}$. The damping $\Delta s_{21}$ over the whole frequency range $0.7-10.0 \mathrm{GHz}$ in $\mathrm{VV}$ polarization is shown in Fig. 9. All curves show very similar transmission characteristics at frequencies below $1 \mathrm{GHz}$. Beginning at this 


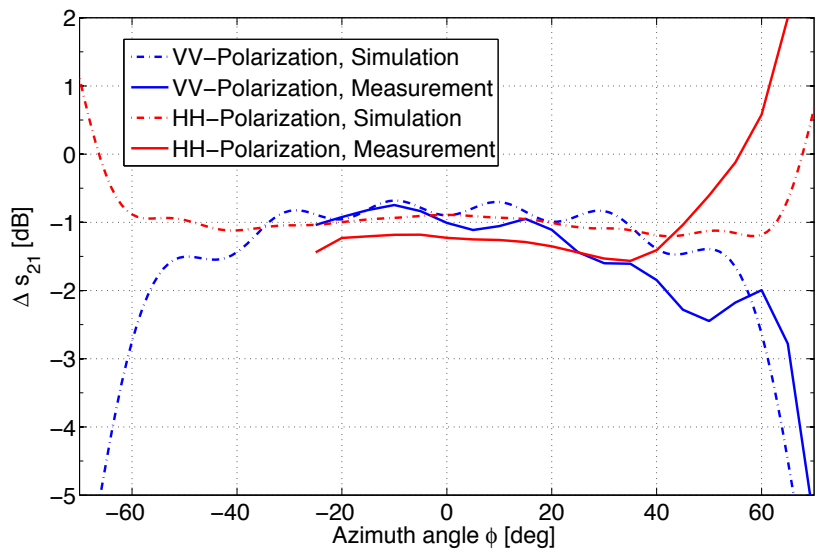

Fig. 7. Damping $\Delta s_{21}$ caused by the wooden slab at $f=1 \mathrm{GHz}$ and the distance $d_{2}=2.70 \mathrm{~m}$ between slab and observing point. Comparison of simulations and measurements in VV and $\mathrm{HH}$ polarization.

frequency the curves start deviating from each other. At this frequency, the free-space wavelength $\lambda_{0}$ is in the range of $30 \mathrm{~cm}$. During its propagation through the material it reduces to approximately $23 \mathrm{~cm}$ due to Eq. (2). At the same time the brick's biggest cavity has a diagonal length of $\approx 11 \mathrm{~cm}$ : this length is in the same range as the wavelength in the material. Thereby the cavity is now visible to the wave, causing electromagnetic interaction and causing scattering. The differences between the curves show the sensitivity of the transmission TTW regarding the positioning of the antenna on the wall. The results for the three positions differ among each other. At $f=2.5 \mathrm{GHz}$, transmission TTW at antenna position B increases to $s_{21, B} \approx+4 \mathrm{~dB}$, whereas the transmission at position $\mathrm{C}$ decreases drastically $s_{21, C} \approx-30 \mathrm{~dB}$. These deviations are due to the interference of wave fractions reflected on the cavities: at the observed point the total electric field is measured and therefore the in-phase sum of all waves might sum up to almost zero. By changing the position of the antenna, the distances to the cavities change accordingly and with them the phases of the partial waves. As the consequence, the transmission shows different results for different antenna positions. In the case of antenna position B and C, $\Delta s_{21}$ can reach values greater than zero. So even focusing effects at certain positions and certain frequencies are possible. It can also be observed that one of the curves always has an amplitude $\Delta s_{21}>-10 \mathrm{~dB}$. This is a hint that many antenna positions can complement each other and the probability of higher $\Delta s_{21}$ increases with the growing number of used antennas. As the transmission is very much dominated by other effects, $\phi_{\text {offset }}$ is negligible.

In the second step, measured data are compared at one single frequency $f=2 \mathrm{GHz}$ in dependence of the azimuth angle $\phi$. Both simulation and measurement data are shown in Fig. 10. As stated in Table 1, simulations were carried out for

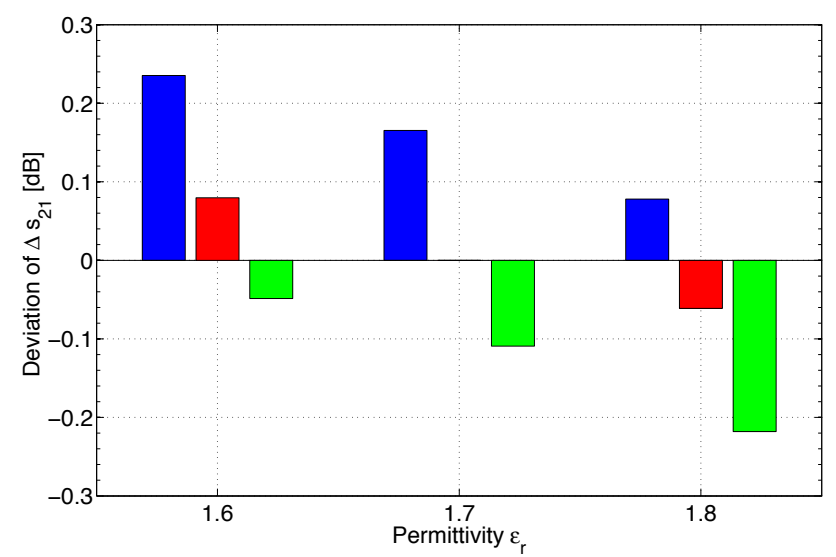

Fig. 8. Simulation of the transmission through the wooden slab with different material parameters (Table 1). Comparison of the deviation in damping $\Delta s_{21}$ at $\phi=0^{\circ}, \theta=0^{\circ}$ with the reference deviation $\varepsilon_{\mathrm{r}}=1.7, \tan \delta=0.07$. The bars represent $\tan \delta=0.06,0.07,0.08$ (blue, red and green respectively).

two different spacing values between the bricks $(g=1 \mathrm{~cm}$ and $2 \mathrm{~cm}$ ). The curves are normalized in such a way that straight radiation loss is coded in the $\phi=0^{\circ}$ value of each curve. Big differences between the simulation and measurement curves can be observed. In the simulations, against prediction, transmission for the bigger gap between the bricks $g=2 \mathrm{~cm}$ lead to bigger damping $\Delta s_{21}$, even though the antenna is directly in front of the gap. Assumptions based on Fig. 9 and the highly sensitive transmission depending on geometry and frequency would require the radiation pattern to be similarly turbulent. As the simulations were carried out for a dense angular step $\Delta \phi=1^{\circ}$, the smoothness of the curves are truthful. This is a result of the perfect periodicity and geometry of the simulation setup. In contrast, the measurements were recorded in sparse angular steps $\Delta \phi=10^{\circ}$. The smoothness of the curves here can be a result of numerical interpolation, hiding local drops in field strength. In spite, the main radiation beam of the antenna is preserved.

As a result, making conclusions from measured reflections to the original objects are not possible as the damping caused by the wall is unpredictable as a high grade of determination is demanded. This includes the exact positions of the bricks and their detailed characteristics, which is practically impossible. Floquet mode analysis and measurement in (Honcharenko et al., 1994) on concrete cinder blocks that have a simpler cross-section and periodicity than brick showed the difficulties of accurate prediction of TTW transmission, revealing the strong dependence of diffraction on frequency and periodicity. Here, the manifold periodicity of the brick wall further increases the difficulty of analysis. The additional dependence on the positioning of the transmitting antenna makes the uniform illumination of the room impossible. This can cause problems in detection and classification 


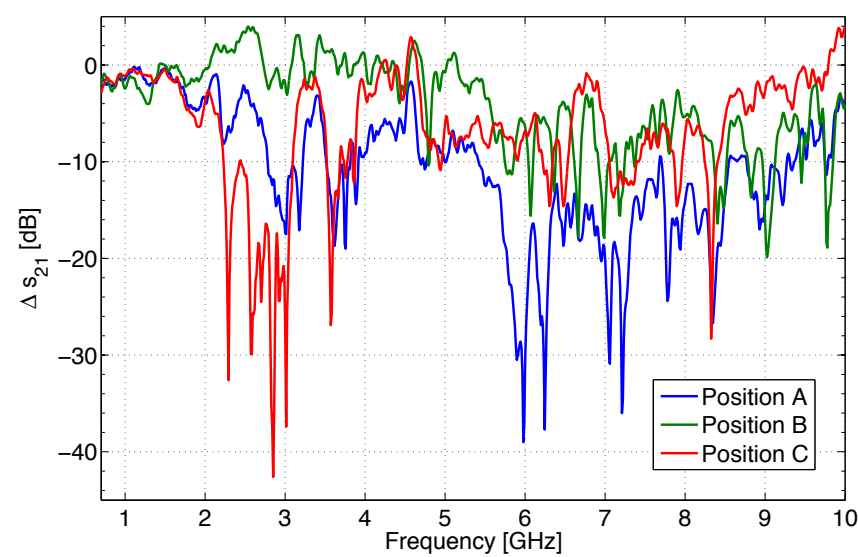

Fig. 9. Damping through brick wall at three different measurement points A, B, C (Fig. 5) with rotation angle $\phi=0^{\circ}$ for the frequency spectrum $0.7-10.0 \mathrm{GHz}$ in $\mathrm{VV}$ polarization.

of scatterers. In the worst case the radar system will be blind to several spots and will not be able detecting anything located there. Together with ultra-wideband (UWB) systems, multi-view approaches are most suitable for solving the problem of incalculable transmission: as the antennas are placed or shifted at different locations, diversities in signal strength get balanced through statistical variation. In the later image fusion process, superposition of the multiple view data will lead to a balance for all scatterers inside the room. The measurements have shown that the unreliable transmission at the different measurement positions is compensated by the increasing probability that one of the measurement positions will cause sufficient transmission. Here with three different measurement positions, there is always one $\Delta s_{21}>-10 \mathrm{~dB}$. With an increasing number of used antennas it is being expected that the probability of higher signal strength TTW increases as well.

\subsection{Inhomogeneous multilayer wall}

The result of the damping measurement through the inhomogeneous multilayer wall over the frequency $0.7-4.3 \mathrm{GHz}$ in VV polarization is shown in Fig. 11. For the calculation of the net damping $\Delta s_{21}$, free-space measurements in the anechoic chamber were used as the reference rather than those in the open test site. As free-space measurements in the anechoic chamber at the required distance $d_{4}=3.00 \mathrm{~m}$ were not available, a quadratic interpolation between the available data for the distances $1.47 \mathrm{~m}, 2.67 \mathrm{~m}$ and $4.14 \mathrm{~m}$ was executed. The mistake committed with this procedure is below $1 \mathrm{~dB}$, which is insignificant to the result. The blue graph represents the measured net loss, the red graph is a linear fit. It indicates that the loss increases approximately with $16 \mathrm{~dB} / \mathrm{GHz}$. For frequencies $f>3.35 \mathrm{GHz}$ the signal falls below the noise level and data are not usable anymore.

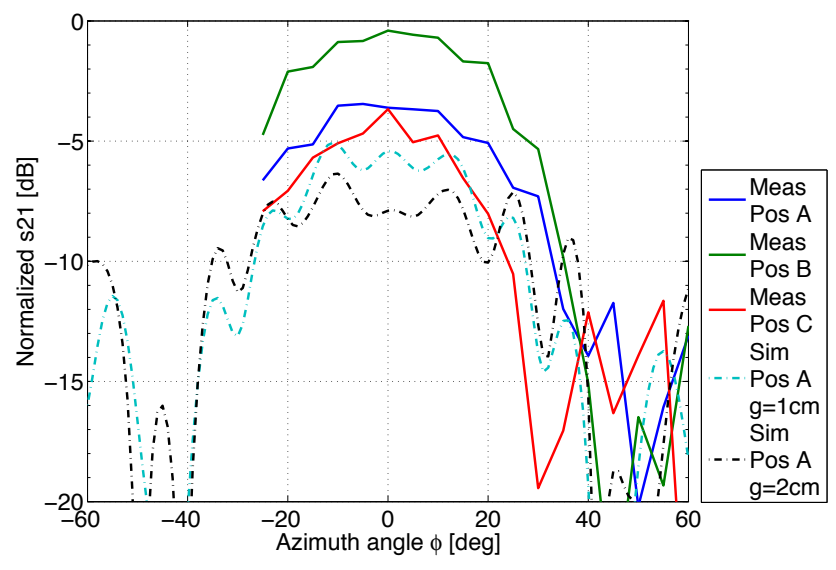

Fig. 10. Radiation pattern of brick wall for $f=2 \mathrm{GHz}$ in VV polarization: comparison of measurement results in positions $\mathrm{A}, \mathrm{B}, \mathrm{C}$ and simulation results for different $\mathrm{H}$ and $\mathrm{V}$ brick spacings $g$.

The radiation patterns in VV polarization for different frequencies up to a maximum of $f=3.3 \mathrm{GHz}$ are shown in Fig. 12. For comparison, measurement data of the freestanding antenna are shown as well. To demonstrate the relative amplitude difference in dependence of the frequency, the curves are normalized equally as in Figure 10: the central radiation direction $\phi=0^{\circ}$ indicates the net loss $\Delta s_{21}$ due to the wall's presence. In the case of the free-standing antenna, net loss is $0 \mathrm{~dB}$. In theory, these values for the damping should agree with the data presented in Fig. 11. Practically, the discrepancies show that the recorded transmission at two different distances $\left(d_{4}=3.00 \mathrm{~m}\right.$ and $\left.d_{1}=1.50 \mathrm{~m}\right)$ can be different, an indication that wave interference plays an important role. The radiation patterns of the antenna in free-space configuration and the antenna on the wall at $0.7 \mathrm{GHz}$ show symmetry. The curves for the antenna on wall at higher frequencies do not show symmetry anymore. The transmission values seem to be randomly distributed within specific tolerances that increase with higher frequency, forming an approximately $12 \mathrm{~dB}$ band in the case of $f=3.3 \mathrm{GHz}$. Preference in radiation direction as shown for the antenna on brick wall vanishes. This and higher sensitivity with increasing frequency show that wave interference of the diffracted waves on the cavities dominates transmission TTW. In addition, the high damping $\Delta s_{21}$ of the walls codetermine the transmission as visible from the amplitude distances between the individual curves.

Very similar to the sole brick wall, the field distribution behind the wall cannot be analyzed analytically nor by simulations as a high degree of determination for the wall is necessary. Sensitivity increases together with the frequency, depending on too many parameters at the relevant frequencies. Wave interference as well as spatial dispersion is even more dominant for the multilayer wall than for the brick wall as the main radiation direction of the wave vanishes. The high 


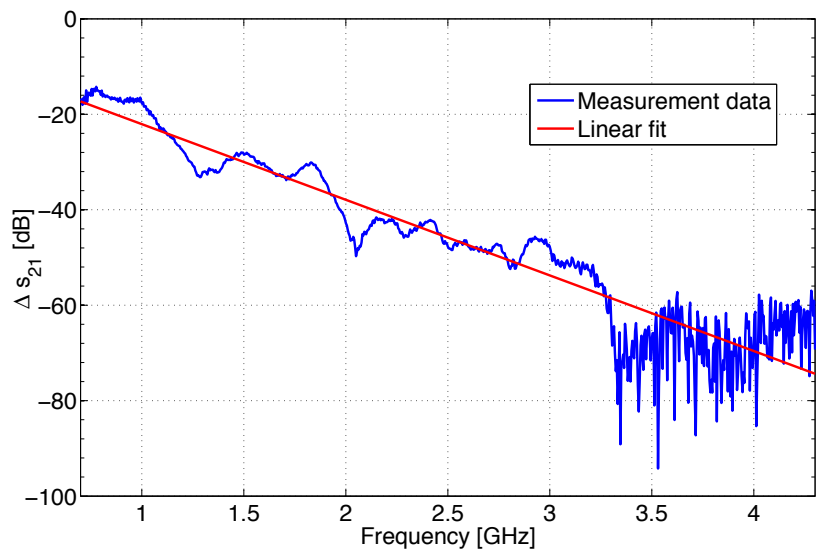

Fig. 11. Damping through multilayer wall in VV polarization for frequency spectrum $0.7-4.3 \mathrm{GHz}$. The linear fit indicates that $\Delta s_{21}$ increases with $16 \mathrm{~dB} / \mathrm{GHz}$. Measurement in anechoic chamber used as reference. Distance between antennas $d_{4}=3.00 \mathrm{~m}$.

damping value of the wall also plays a very important role. As the signal needs to travel back and forth, high dynamic range of the imaging system is required.

\section{Conclusions}

In this work, simulations and measurements for the determination of near-field radiation patterns and their distortion by three different kinds of wall have been presented.

It has been shown that simulations and measurements are in very good agreement in case of the homogeneous wooden slab, making transmission through a homogeneous slab predictable. Sensitivity analysis for different material properties have shown that the error range due to imprecise material knowledge is much smaller than the error range of the material properties taken as a basis in this work. Additionally they are within measurement inaccuracies and therefore could be neglected. This shows that simulations are reliable and can be used in the prediction of transmission TTW. In the case of the inhomogeneous brick wall, three different measurement positions lead to different near-field patterns. The results for the TTW transmission in normal incidence show the sensitivity of the brick wall both on the positioning of the AUT and on frequency: depending on these variables it is possible that the potential TWRI system is blind to certain spots. This is caused by interference of waves scattered at the cavities. Nevertheless, the main lobe of the radiation pattern is conserved qualitatively. This does not apply for the inhomogeneous multilayer wall: wave interference caused by the cavities garbles the radiation pattern in such a way that field distribution seems to be randomly distributed within certain aberrations, even destructing the symmetry of the radiation pattern. In addition, transmission is dominated by the high damping values. The field strength measured behind walls

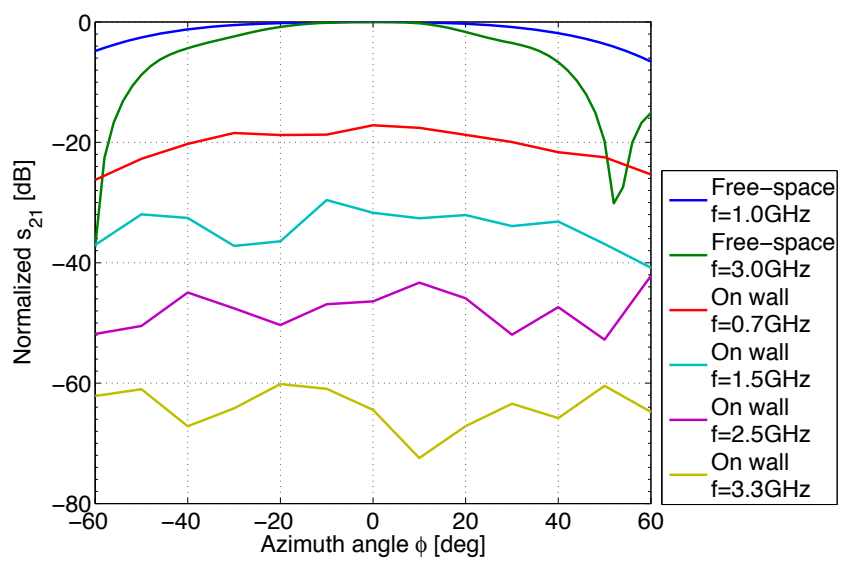

Fig. 12. Distorted radiation pattern of antenna on inhomogeneous multilayer wall in comparison to radiation pattern of free-standing antenna at different frequencies in VV polarization. Distance between antennas $d_{1}=1.50 \mathrm{~m}$.

made of inhomogeneous material depends on many variables like frequency, position of the AUT, direction of radiation and the position of investigation. Simulations are not reliable because the determination of the whole wall is essential. As similar characteristics were reported for reinforced concrete walls (Dalke et al., 2000; Antonini et al., 2003), this problem spans the vast majority of wall structures.

The appearances of unpredictable signal nulls in the frequency spectrum make the use of narrowband single-view systems inadequate. Multi-view approaches (e.g. multi-static radar, MIMO, SAR) based on UWB architecture can solve the problem of unreliable transmission by statistically balancing the field distribution behind the wall.

Acknowledgements. This work was supported under grant number Aramis $041-19$ by Armasuisse $W+T$, administrational agency of the Swiss Federal Department of Defense, Civil Protection and Sports.

\section{References}

Ahmad, F., Amin, M. G., and Mandapati, G.: Autofocusing of Through-the-Wall Radar Imagery Under Unknown Wall Characteristics, IEEE T. Image. Process., 16, 1785-1795, doi:10.1109/TIP.2007.899030, 2007.

Amin, M. G.: Through-the-Wall Radar Imaging, CRC Press, Boca Raton (FL), USA, 2011.

Antonini, G., Orlandi, A., and D'elia, S.: Shielding Effects of Reinforced Concrete Structures to Electromagnetic Fields due to GSM and UMTS Systems, IEEE T. Magn. , 39, 1582-1585, doi:10.1109/TMAG.2003.810327, 2003.

Balanis, C. A.: Antenna theory: analysis and design, 3 Edn, John Wiley \& Sons, Hoboken (NJ), USA, 2005.

Baranoski, Edward J.: Through-wall imaging: Historical perspective and future directions, J. Frankl. Inst., 345, 556-569, doi:10.1016/j.jfranklin.2008.01.005, 2008. 
Chang, P. C., Burkholder, R. J., and Volakis, J. L.: Adaptive CLEAN With Target Refocusing for Through-Wall Image Improvement, IEEE T. Antenn. Propag., 58, 155-162, doi:10.1109/TAP.2009.2036131, 2010.

Dalke, R. A., Holloway, C. L., McKenna, P., Johansson, M., and Ali, A. S.: Effects of Reinforced Concrete Structures on RF Communications, IEEE T. Electromagn. C., 42, 486-496, doi:10.1109/15.902318, 2000.

Edgar, D.: HFSS 13: Hybrid FE-BI for Efficient Simulation of Radiation and Scattering, Ansys. Inc., 2011.

ETS-Lindgren: Model 3164 Series - Open Boundary Quad-Ridged Horns - User Manual, 2010.

Farwell, M., Ross, J., Luttrell, R., Cohen, D., Chin, W., and Dogaru, T.: Sense through the wall system development and design considerations, J. Frankl. Inst., 345, 570-591, ISSN 0016-0032, doi10.1016/j.jfranklin.2008.01.004, 2008.

Ferris Jr, D. D. and Currie, N. C.: Survey of current technologies for through-the-wall surveillance (TWS), P. SPIE., 3577, 62-72, 1999.

Honcharenko, W. and Bertoni, H.: Transmission and Reflection Characteristics at Concrete Block Walls in the UHF Band Proposed for Future PCS, IEEE T. Antenn. Propag., 42, 232-239, 1994.

Jia, Y., Kong, L., and Yang, X.: A novel approach to target localization through unknown walls for through-the-wall radar imaging, Progr. Electromagn. Res., 119, 107-132, 2011.
Li, L., Zhang, W., and Li, F.: A Novel Autofocusing Approach for Real-Time Through-Wall Imaging Under Unknown Wall Characteristics, IEEE T. Geosci. Remote, 48, 423-431, doi:10.1109/TGRS.2009.2024686, 2010.

Muqaibel, A. H.: Characterization of Ultra Wideband Communication Channels, Ph.D. thesis, Virginia Polytechnic Institute and State University, Blacksburg (VA), USA, 2003.

Seybold, J. S.: Introduction to RF Propagation, John Wiley \& Sons, Hoboken (NJ), USA, 2005.

Sheen, D. M., McMakin, D. L., and Hall, T. E.: Near Field Imaging at Microwave and Millimeter Wave Frequencies, IEEE/MTTS International Microwave Symposium, Honolulu (HI), USA, 3-8 June 2007, 693-1696, doi:10.1109/MWSYM.2007.380033, 2007.

Stavrou, S. and Saunders, S. R.: Review of constitutive parameters of building materials, in: Proceedings of the 12th International Conference on Antennas and Propagation ICAP, Exeter, UK, 31 March - 3 April 2003, 1, 211-215, doi:10.1049/cp:20030052, 2003.

Thajudeen, C., Hoorfar, A., Ahmad, F., and Dogaru, T.: Measured complex permittivity of walls with different hydration levels and the effect on power estimation of twri target returns, Prog. Electromagn. Res. B, 30, 177-199, doi:10.2528/PIERB10091004, 2011.

Wang, G. and Amin, M. G.: Imaging Through Unknown Walls Using Different Standoff Distances, IEEE T. Signal Proces., 54, 4015-4025, 2006. 